\title{
PERFORMANCE OF MMSE STBC MC-CDMA OVER RAYLEIGH AND MIMO METRA CHANNELS
}

\author{
J-M. AUFFRAY, J-Y. BAUDAIS, J-F. HELARD
}

IETR / INSA - 20 avenue des Buttes de Coësmes, 35043 RENNES - FRANCE

\begin{abstract}
The performance of MMSE Single-user Detection (SD) and Multi-user Detection (MD) STBC MC-CDMA systems are analysed and compared in the case of two transmit antennas and one or two receive antennas over Rayleigh fading channels and then over the stochastic MIMO METRA channel model. With two transmit and one receive antennas, MD achieves a gain of roughly $1 \mathrm{~dB}$ for non-full load systems while the same performance are obtained with MD and SD for full load systems. Besides, with two receive antennas, we present a sub-optimal and an optimal MMSE SD MIMO MC-CDMA schemes, this last one offering a very good performance/complexity trade-off. Finally, the very good behaviour of MMSE STBC MC-CDMA systems is confirmed over the realistic METRA MIMO channel.
\end{abstract}

\section{INTRODUCTION}

Nowadays, Multi-Carrier Code Division Multiple Access (MC-CDMA) is the most promising candidate for the air interface downlink of the $4^{\text {th }}$ Generation mobile radio systems. MC-CDMA combines the robustness of Orthogonal Frequency Division Multiplex (OFDM) modulation with the flexibility of CDMA [1]. On the other hand, Multiple Input Multiple Output (MIMO) communication systems, by using several antennas at the transmitter and at the receiver, inherit space diversity to mitigate fading effects. When the channel is not known at the transmitter, taking benefit of the transmit diversity requires methods such as space-time coding which uses coding across antennas and time [2]. For example, Space-Time Block Coding (STBC), as proposed by Alamouti in [3] and Tarokh in [4], provides full spatial diversity gains, no intersymbol interference and low complexity ML receiver if transmission matrix is orthogonal. Moreover with STBC, only one receive antenna can be used, leading in that case to MISO (Multiple Input Single Output) systems.

In [5], it has been shown that unity-rate Alamouti's STBC QPSK MC-CDMA outperforms half-rate Tarokh's STBC 16-QAM MC-CDMA, while offering the same effective throughput of $2 \mathrm{bit} / \mathrm{s} / \mathrm{Hz}$ without channel coding. Indeed, in order to maintain the same effective throughput, half-rate STBC codes have to be employed in conjunction with higher modulation schemes as 16-QAM, which are more prone to errors and hence degrade the performance of the system. Moreover, unity-rate 
STBC code combined with channel-coded schemes as turbo-codes provides substantial performance improvement over the non-unity-rate STBC. Hence for the same effective throughput, the reduction in coding rate is best invested in turbocodes, rather than STBC.

In this paper, we compare in the downlink case and without channel coding the performance of Alamouti's STBC MC-CDMA systems combined with Multi-user Detection (MD) or Single-user Detection (SD) schemes. For this comparison, the considered detection schemes are based on Mean Square Error (MSE) criterion, since MMSE detection is known as the most efficient SD technique [6]. In order to obtain asymptotic performance, the algorithms are evaluated over Rayleigh fading channels in the first part. Then, some further results are given over the more realistic stochastic MIMO channel model developped within the European IST METRA (Multi Element Transmit Receive Antennas) project.

\section{SYSTEM DESCRIPTION}

Figure 1 shows the considered MIMO MC-CDMA system for the $j^{\text {th }}$ user based on Alamouti's STBC with $N_{t}=2$ transmit antennas and $N_{r}=2$ receive antennas [3]. Each user $j$ transmits simultanously from the two antennas the symbol $x_{j}^{0}$ and $x_{j}^{1}$ at time $t$, and the symbols $-x_{j}{ }^{*}$ and $x_{j}{ }^{0^{*}}$ at time $t+T_{x}$ where $T_{x}$ is the OFDM symbol duration. At the output of the space-time encoder, the data symbols $\mathbf{x}^{0}=\left[x_{1}{ }^{0} \ldots x_{j}^{0} \ldots x_{N u}{ }^{0}\right]^{T}$ of the $N_{u}$ users are multiplied by their specific orthogonal Walsh-Hadamard (W-H) spreading code $\mathbf{c}_{j}=\left[c_{j, 1} \ldots c_{j, k} \ldots c_{j, L c}\right]^{T}$ where $c_{j, k}$ is the $k^{\text {th }}$ chip, and [.] $]^{T}$ denotes matrix transposition (the same goes for symbol 1). $\mathbf{c}_{j}$ is the $j^{\text {th }}$ column vector of the $L_{c} \times N_{u}$ spreading code matrix C. In this paper, the length $L_{c}$ of the spreading sequences is equal to the number $N_{c}$ of subcarriers and to the maximum number $N_{u}$ of simultaneous active users in the full-load case. Each data symbol $x_{j}$ is then transmitted in parallel on $N_{c}$ QPSK modulated subcarriers. The vector obtained at the $r^{\text {th }}$ receive antenna after the OFDM demodulation and deinterleaving, at time $t$ and $t+T_{x}$, is given by:

$$
\boldsymbol{R}_{r}=\boldsymbol{H}_{r} \boldsymbol{C X}+\boldsymbol{N}_{r} \quad \text { with } \quad \boldsymbol{H}_{r}=\left[\begin{array}{cc}
\mathbf{H}_{l r} & \mathbf{H}_{2 r} \\
\mathbf{H}_{2 r}^{*} & -\mathbf{H}_{l r}^{*}
\end{array}\right]
$$

where $\boldsymbol{R}_{r}=\left[\mathbf{r}_{r}^{\mathrm{T}}(t) \mathbf{r}_{r}^{H}\left(t+T_{x}\right)\right]^{T}$ with $\mathbf{r}_{r}(t)=\left[r_{r, l}(t) \ldots r_{r, k}(t) \ldots r_{r, N c}(t)\right]^{T}$ the vector of the $N_{c}$ received signals at time $t$ and [.] ${ }^{H}$ denotes the Hermitian transpose (or conjugate transpose), $\mathbf{H}_{t r}=\operatorname{diag}\left\{h_{t r, l}, \ldots, h_{t r, N c}\right\}(t, r \in\{1,2\})$ is a $N_{c}$ x $N_{c}$ diagonal matrix with $h_{t r, k}$ the complex channel frequency response, for the subcarrier $k$ between the transmit antenna $t$ to the receive antenna $r$. Time invariance during two MC-CDMA symbols are assumed to permit the recombination of symbols when STBC is used. $C$ $=\operatorname{diag}\{\mathbf{C}, \mathbf{C}\}$ with $\mathbf{C}=\left[\mathbf{c}_{l} \ldots \mathbf{c}_{j} \ldots \mathbf{c}_{N u}\right]$ is the $L_{c} \times N_{u}$ matrix of user's spreading codes with the column vector $\mathbf{c}_{j}$ equal to the spreading code of user $j, X=\left[\mathbf{x}^{0 T} \mathbf{x}^{1 T}\right]^{T}$. $\boldsymbol{N}_{r}=\left[\mathbf{n}_{r}{ }^{T}(t) \mathbf{n}_{r}{ }^{H}\left(t+T_{x}\right)\right]^{T}$ with $\mathbf{n}_{r}(t)=\left[n_{r, l}(t) \ldots n_{r, k}(t) \ldots n_{r, N c}(t)\right]^{T}$ is the Additive White Gaussian Noise (AWGN) vector with $n_{r, k}(t)$ representing the noise term at subcarrier $k$, for the $r^{\text {th }}$ receive antenna at time $t$ with variance given by $\sigma_{k}^{2}=\mathrm{E}\left\{\left|n_{k}\right|^{2}\right\}=N_{0} \forall k$. 


\section{Performance Of Mmse StbC MC-CDMA Over Rayleigh AND MiMO METRA CHANNELS}
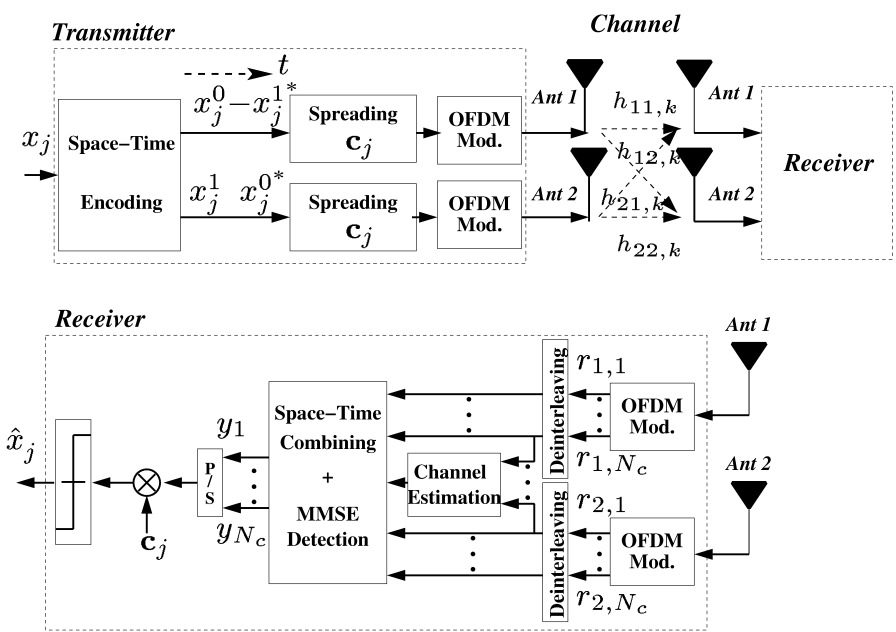

Figure 1. MC-CDMA transmitter and receiver for user $j$ with transmit and receive diversity.

\section{MULTI-USER DETECTION VERSUS SINGLE-USER DETECTION IN THE MIMO AND MISO CASES}

In the receiver, in order to detect the two transmitted symbols $x_{j}^{0}$ and $x_{j}{ }^{1}$ for the desired user $j, \mathrm{SD}$ or MD detection schemes based on the MSE criterion are applied to the received signals in conjunction with STBC decoding. In the SISO case, it has been shown in [6] that MMSE SD is the most efficient SD scheme, while MMSE $\mathrm{MD}$, also called Global-MMSE, is optimal according to the MSE criterion for any number of active users and any power distribution [7]. Here we compare in the MISO case MMSE SD with a new MMSE MD algorithm. Besides, in the MIMO case, an optimal and a sub-optimal MMSE SD algorithms are presented and compared.

\subsection{MMSE Single-user detection in the MISO and MIMO cases}

After equalisation, for each receive antenna $r$, the two successive received signals are combined. The resulting signals from the $N_{r}$ receive antennas are then added to detect the two symbols $x_{j}^{0}$ and $x_{j}{ }^{1}$. After despreading and threshold detection, the detected data symbols $\hat{x}_{j}^{0}$ and $\hat{x}_{j}^{1}$ for user $j$ are:

$$
\left[\begin{array}{ll}
\hat{x}_{j}^{0} & \hat{x}_{j}^{1}
\end{array}\right]^{T}=\left[\begin{array}{ll}
\mathbf{c}_{j}^{T} & \mathbf{c}_{j}^{T}
\end{array}\right] \boldsymbol{Y}=\left[\begin{array}{ll}
\mathbf{c}_{j}^{T} & \mathbf{c}_{j}^{T}
\end{array}\right] \sum_{r=1}^{N r} \boldsymbol{G}_{r} \boldsymbol{R}_{r} \text { with } \boldsymbol{G}_{r}=\left[\begin{array}{cc}
\mathbf{G}_{1 r} & \mathbf{G}_{2 r}^{*} \\
\mathbf{G}_{2 r} & -\mathbf{G}_{1 r}^{*}
\end{array}\right] \text { (2) }
$$


where $\mathbf{G}_{t r}$ is a diagonal matrix containing the equalization coefficients for the channel between the transmit antenna $t$ and the receive antenna $r$. To detect for example $x_{i}^{0}$, the MMSE SD coefficients $g_{t r, k}$ minimises the mean square value of the error $\varepsilon_{k}^{0}$ between the signal $\sum_{i=1}^{N_{u}} c_{i, k} x_{i}^{0}$ transmitted on subcarrier $k$ and the assigned output $y_{k}{ }^{0}$ of the equalizer. Besides, no knowledge of the spreading codes $\mathbf{c}_{i}(i \neq j)$ of the interfering users is required to derive the MMSE SD coefficients.

Table 1. MMSE SD equalization coefficients $g_{t r, k}$ and resulting equalized channel terms $h_{\text {eq, } k}^{0}$ and $h_{\text {eq, }}{ }^{1}$ to detect the symbol $x_{j}^{0}$ for the sub-optimal MMSE(1) SD and optimal MMSE(2) SD

\begin{tabular}{|c|c|c|}
\cline { 2 - 3 } \multicolumn{1}{c|}{} & MMSE(1) SD & MMSE(2) SD \\
\hline $\boldsymbol{g}_{t r, \boldsymbol{k}}$ & $h_{t r, k}^{*} /\left[\sum_{t=1}^{N_{t}}\left|h_{t r, k}\right|^{2}+\frac{1}{\gamma_{r, k}}\right]$ & $h_{t r, k}^{*} /\left[\sum_{t=1}^{N_{t}} \sum_{r=1}^{N_{r}}\left|h_{t r, k}\right|^{2}+\frac{1}{\gamma_{r, k}}\right]$ \\
\hline $\boldsymbol{h}_{\boldsymbol{e q}, \boldsymbol{k}} \mathbf{0}$ & $\sum_{t=1}^{N_{t}} \sum_{r=1}^{N_{r}}\left|h_{t r, k}\right|^{2}$ & $\frac{\sum_{t=1}^{N_{t}} \sum_{r=1}^{N_{r}}\left|h_{t r, k}\right|^{2}}{\sum_{t=1}^{N_{t}}\left|h_{t r, k}\right|^{2}+\frac{1}{\gamma_{r, k}}}$ \\
\hline $\boldsymbol{h}_{\text {eq, } \boldsymbol{k}}^{\mathbf{1}}$ & 0 & $\sum_{t=1}^{N_{t}}\left|h_{t r, k}\right|^{2}+\frac{1}{\gamma_{r, k}}$ \\
\hline
\end{tabular}

Table 1 gives the MMSE SD equalization coefficients $g_{t r, k}$ and the resulting equalized channel coefficients $h_{e q, k}{ }^{0}$ and $h_{e q, k}{ }^{1}$ to detect $x_{i}^{0}$ and $x_{i}^{1}$ respectively. For the optimal MMSE(2) SD algorithm, $N_{t} N_{r}$ channel coefficients $h_{t r, k}$ are taken into account, while only $N_{t}$ are considered for $\operatorname{MMSE}(1) \mathrm{SD}$ algorithm. Thus, an excessive noise amplification for low subcarrier signal to noise ratio $\gamma_{r, k}$ is more unlikely with this new MMSE(2) SD algorithm than with MMSE(1) SD algorithm. In both cases, to detect for example $x_{i}^{0}$, the interference terms generated by $x_{i}^{1}$ are cancelled, i.e., $h_{e q, k}{ }^{1}=0$. On the other hand, for large SNR, MMSE SD restores the orthogonality among users, i.e., $h_{e q, k}{ }^{0}$ tends to one when $\gamma_{r, k}$ increases.

\subsection{MMSE Multi-user detection in the MISO case}

Contrary to MMSE SD, MD is carried out by exploiting the knowledge of the spreading codes $\mathbf{c}_{i}(i \neq j)$ of the interfering users. As the optimum Maximum Likelihood (ML) detector is too complex, we consider here sub-optimal linear MMSE MD which is optimal according to the MSE criterion, and applied here for the first time to space-time block coded signals. The MMSE MD technique aims to minimize the mean square error at the input of the threshold detector between the transmitted symbol $x_{j}$ and the estimated one $\hat{x}_{j}$. The two detected data symbol $\hat{x}_{j}^{0}$ and $\hat{x}_{j}^{1}$ for user $j$ are:

$$
\left[\begin{array}{ll}
\hat{x}_{j, o p t}^{0} & \hat{x}_{j, o p t}^{1}
\end{array}\right]^{T}=\mathbf{W}_{j, o p t}^{H} \boldsymbol{R}=\left[\begin{array}{ll}
\mathbf{c}_{j}^{T} & \mathbf{c}_{j}^{T}
\end{array}\right] \boldsymbol{G}_{r}^{H} \boldsymbol{R}=\left[\begin{array}{c}
\mathbf{W}_{j, o p t}^{0 H} \\
\mathbf{W}_{j, o p t}^{1 H}
\end{array}\right]\left[\begin{array}{c}
\mathbf{r}_{r}(t) \\
\mathbf{r}_{r}^{*}\left(t+T_{x}\right)
\end{array}\right]
$$




\section{Performance Of Mmse Stbc Mc-CDMA Over Rayleigh And MiMO METRA CHANNELS}

where $\mathbf{W}_{j, \text { opt }}{ }^{H}$ is the optimal $2 \times 2 \mathrm{~L}_{\mathrm{c}}$ weighting matrix and $\boldsymbol{G}_{r}{ }^{H}$ is the equalization coefficient matrix of the MISO channel at the antenna $r$. According to the Wiener filtering, the optimal weighting matrix is the matrix which minimises the mean square error $\mathrm{E}\left|\mathbf{W}_{j}^{H} \boldsymbol{R}-\left[x_{j}^{0} x_{j}^{1}\right]^{T}\right|^{2}$. The $2 L_{c}$ weighting vectors $\mathbf{w}_{j, \text { opt }}{ }^{0}$ and $\mathbf{w}_{j, \text { opt }}{ }^{1}$ to detect $x_{j}^{0}$ and $x_{j}^{1}$ respectively are equal to:

$$
\mathbf{W}_{j, \text { opt }}^{0}=\Gamma_{\boldsymbol{R}, \boldsymbol{R}}^{-1} \Gamma_{\boldsymbol{R}, x_{j}^{0}} \quad \mathbf{W}_{j, \text { opt }}^{1}=\Gamma_{\boldsymbol{R}, \boldsymbol{R}}^{-1} \Gamma_{\boldsymbol{R}, x_{j}^{1}}
$$

where $\Gamma_{\boldsymbol{R}, \boldsymbol{R}}$ is the autocorrelation matrix of the received vector $\boldsymbol{R}$ and $\Gamma_{\boldsymbol{R}}, x_{j}^{0}$ and $\Gamma_{\boldsymbol{R}}, x_{j}^{1}$ are the cross-correlation vector between the received signal vector $\boldsymbol{R}$ and the desired symbol $x_{j}^{0}$ and $x_{j}^{1}$ respectively. Hence the optimal weighting matrix is:

$$
\mathbf{W}_{j, o p t}^{H}=E_{s}\left[\mathbf{c}_{j}^{T} \mathbf{c}_{j}^{T}\right] \boldsymbol{H}^{H}\left(\boldsymbol{H} \boldsymbol{C} \boldsymbol{\Gamma}_{\boldsymbol{X}, \boldsymbol{X}} \boldsymbol{C}^{T} \boldsymbol{H}^{H}+\Gamma_{\boldsymbol{N}, \boldsymbol{N}}\right)^{-1}
$$

where $\boldsymbol{E}_{\boldsymbol{S}}=\mathrm{E}\left|x_{j}\right|^{2}, \Gamma_{\boldsymbol{X}, \boldsymbol{X}}$ is the autocorrelation matrix of the transmitted symbols vectors $\boldsymbol{X}, \Gamma_{\boldsymbol{N}, \boldsymbol{N}}$ is the autocorrelation matrix of the noise vector $\boldsymbol{N}$.

In the full load case $\left(N_{u}=L_{c}\right)$ and only in that case, the four equalization coefficients matrix $\mathbf{G}_{t r}$ included in $\boldsymbol{G}_{r}$ are diagonal matrix with the $k^{\text {th }}$ subcarrier equalization coefficient equal to the coefficient obtained for MMSE SD given in table 1 for $N_{r}=1$. In the non-full load case $\left(N_{u}<L_{c}\right)$, the matrix $\mathbf{G}_{t r}$ are no more diagonal. In that case, the MMSE MD algorithm outperforms the MMSE SD algorithm, since the MMSE MD scheme minimises the decision error taking into account the despreading process instead of minimizing the error independently on each subcarrier.

\section{SIMULATION RESULTS OVER RAYLEIGH FADING CHANNEL}

For these results, frequency non-selective Rayleigh fading per subcarrier and time invariance during two MC-CDMA symbols are assumed to permit the recombination of symbols when STBC is used. Based on these assumptions and considering ideal time and frequency interleaving, the complex channel fading coefficients perfectly estimated are considered uncorrelated for each subcarrier $k$ and mutually independent from each transmit antenna to each receive antenna. With these uncorrelation and independence hypothesis, the asymptotic performance of the studied algorithms can be obtained.

Simulation results are carried out without channel coding to evaluate MMSE MD and MMSE SD performance in the MIMO and MISO cases. The length of the spreading codes $\left(L_{c}=16\right)$ is equal to the number of subcarriers $N_{c}$. Results are first compared in terms of BER performance versus $E_{b} / N_{0}$. The different subcarriers are supposed to be multiplied by independent non-selective Rayleigh fading perfectly estimated. It is assumed that all the users' signals are received with the same mean power. We do not take into account the power gain provided by the use of multiple antennas, i.e. only the spatial diversity gain is taken into account. The Matched 
Filter (MF) bound is given as reference as well as the performance of the MCCDMA system combined with MMSE SD or GMMSE in the SISO case.

\subsection{Full load system}

Figure 2 shows the performance of MMSE MD and MMSE SD with and without transmit and receive diversity, with full-load systems for $L_{c}=N_{c}=N_{u}=16$. As expected, the performance of MC-CDMA is highly improved when combined with STBC in order to exploit the transmit diversity, and when multiple receive antennas are used in order to take benefit of receive diversity. Moreover, in the MISO case $\left(N_{t} N_{r}=2-1\right)$, the performance of 21 MMSE MD and 21 MMSE SD are the same because the equalization coefficient matrix $\mathbf{G}_{t r}$ is a diagonal matrix with the $k^{t h}$ subcarrier equalization coefficient equal to the coefficient obtained for MMSE SD. Besides, 22 MMSE SD system $\left(N_{t} N_{r}=2-2\right)$ is the best scheme and offers a very good performance/complexity trade-off.

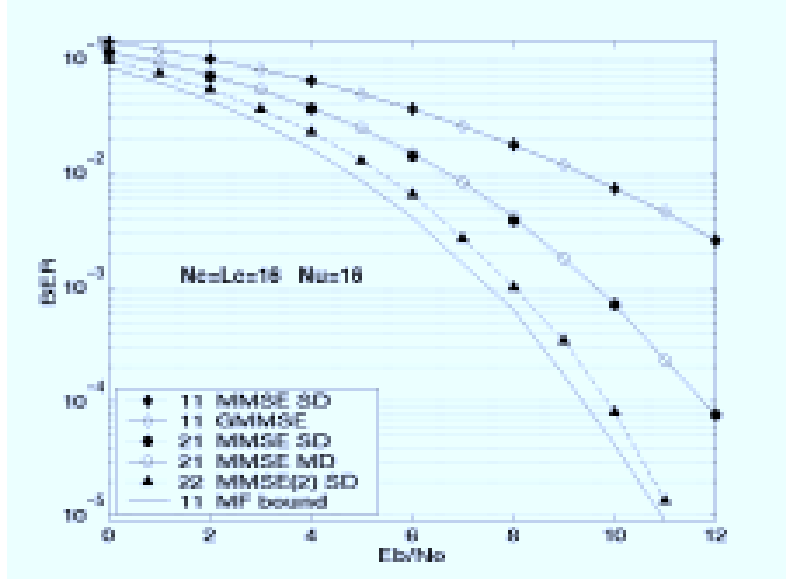

Figure 2. Performance of MMSE MD and SD over Rayleigh fading channels for SISO $\left(N_{t} N_{r}=11\right)$, MISO $\left(N_{t} N_{r}=21\right)$ and MIMO $\left(N_{t} N_{r}=22\right)$ systems with $N_{c}=L_{c}=16$. $N_{u}=16$ (full load).

\subsection{Half load system}

The performance of MMSE MD and MMSE SD with and without transmit and receive diversity, with half-load systems for $L_{c}=N_{c}=16$ and $N_{u}=8$ are presented figure 3. With two transmit antennas and one receive antenna, 21 MMSE MD achieves a gain of roughly $1 \mathrm{~dB}$ compared to 21 MMSE SD at high SNR. Again, 22 MMSE SD system is the best scheme even if the gain compared to other systems is lower than in the full load case. 


\section{Performance Of Mmse Stbc Mc-CDMA Over Rayleigh And MiMO METRA CHANNELS}

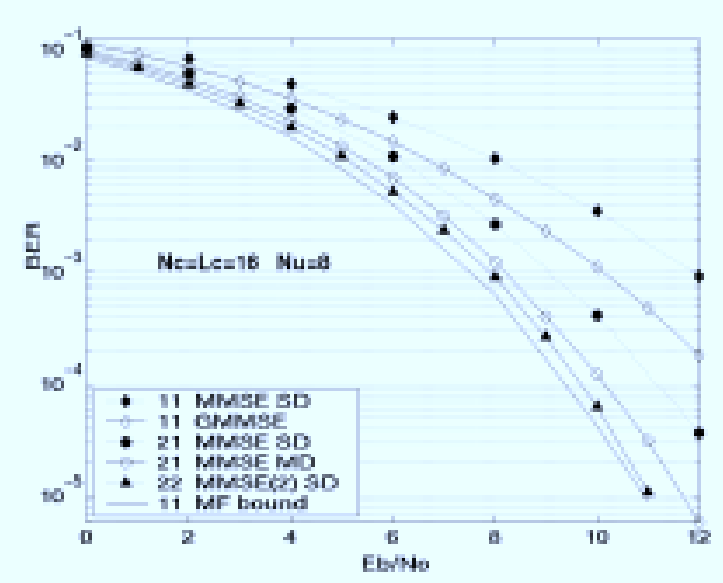

Figure 3. Performance of MMSE MD and SD over Rayleigh fading channels for SISO $\left(N_{t} N_{r}=11\right)$, MISO $\left(N_{t} N_{r}=21\right)$ and MIMO $\left(N_{t} N_{r}=22\right)$ systems with $N_{c}=L_{c}=16$. $N_{u}=8$ (half load).

\subsection{Performance versus system load}

Finally, in figure 4, the performance of sub-optimal $\operatorname{MMSE}(1)$ and optimal MMSE(2) SD MIMO MC-CDMA are compared to MMSE MD MISO MC-CDMA and MMSE SD SISO MC-CDMA. The maximum number $N_{u}$ of active users versus the required $E_{b} / N_{0}$ to achieve a $\mathrm{BER}=10^{-3}$ is given for $L_{c}=N_{c}=16$ and equal mean power signals.

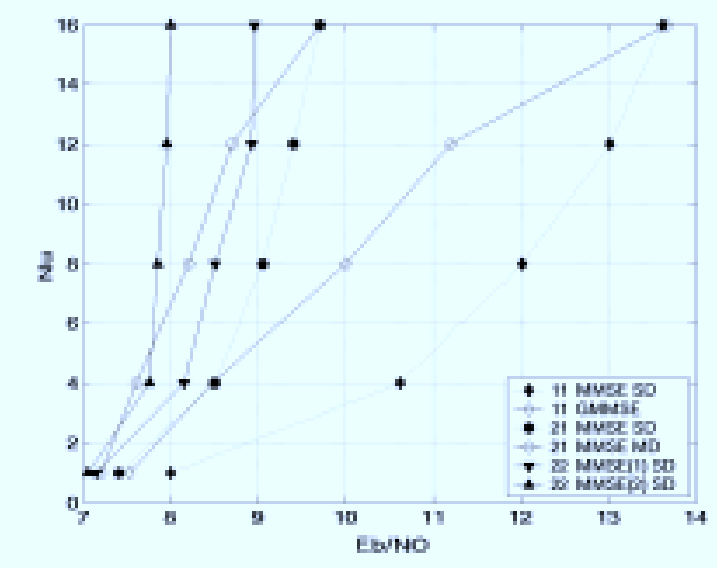

Figure 4. Number $N_{u}$ of active users versus the required $E_{b} / N_{0}$ to achieve a $B E R=10^{-3}$ with MMSE MD and SD over Rayleigh fading channels for SISO $\left(N_{t} N_{r}=11\right)$, MISO $\left(N_{t} N_{r}=21\right)$ and MIMO $\left(N_{t} N_{r}=22\right)$ systems; $N_{c}=L_{c}=16$. 
For non full load cases, the gain of MMSE MD compared to MMSE SD, which is roughly equal to $2 \mathrm{~dB}$ for SISO systems, decreases to less than $1 \mathrm{~dB}$ for MISO systems. Furthermore, the most important result is that, for any load, the low complex and new 22 MMSE(2) SD MIMO scheme outperforms all studied MD and SD MISO and SISO systems. Furthermore, the spatial diversity gain is all the more significant when the number of active users $N_{u}$ is high.

\section{SIMULATION RESULTS OVER METRA CHANNEL}

A major characteristic of the stochastic MIMO channel model developed within the European Union IST research METRA project is that, contrary to other directional models, it does not rely on a geometrical description of the environment under study [8]. It is a complex Single-Input Single-Output (SISO) Finite Impulse Response (FIR) filter whose taps are computed so as to simulate time dispersion, fading and spatial correlation. To simulate MIMO radio channels, it has to be inserted between a parallel-to-serial and serial-to-parallel converters. Besides, the correlation properties in the spatial domain of the MIMO radio channel are obtained by the Kronecker product of two independent correlation matrices defining the correlation properties at the Base Station (BS) and Mobile Station (MS).

Table 2. Main system and MIMO channel parameters

\begin{tabular}{|l|c|l|c|}
\hline OFDM symbol duration $(\mu \mathrm{s})$ & 3.2 & Number $N_{c}$ of subcarriers & 64 \\
\hline Guard interval $(\mu \mathrm{s})$ & 0.5 & Length $L_{c}$ of the spreading codes & 64 \\
\hline Center frequency $(\mathrm{GHz})$ & 5.2 & Signal Bandwidth $(\mathrm{MHz})$ & 20 \\
\hline Channel Profile & BRAN A & Velocity $(\mathrm{km} / \mathrm{h})$ & 3.6 \\
\hline Doppler Spectrum & Jakes & Measured coherence bandwidth $(\mathrm{MHz})$ & 5.8 \\
\hline Pattern & Omni. & Doppler oversampling factor & 2 \\
\hline DoA azimuth & 0 & Elevation angle $(\mathrm{deg})$ & 90 \\
\hline
\end{tabular}

Table 2 summarizes the main system and channel parameters. In the correlated MISO and MIMO cases, we consider in the BS and MS an array of 2 uniformly spaced antennas with an inter-element separation fixed to $1.5 \lambda$ and $0.4 \lambda$ respectively. Then, the envelope correlation coefficients between antennas are:

$$
R_{B S}=\left[\begin{array}{cc}
1 & 0.265 \\
0.265 & 1
\end{array}\right] \quad R_{M S}=\left[\begin{array}{cc}
1 & 0.294 \\
0.294 & 1
\end{array}\right]
$$

which have been derived from $4 \times 4$ correlation matrices obtained through experimental measurements in real indoor scenario [8]. 


\section{Performance Of Mmse Stbc Mc-CDMA Over Rayleigh And MiMO METRA CHANNELS}

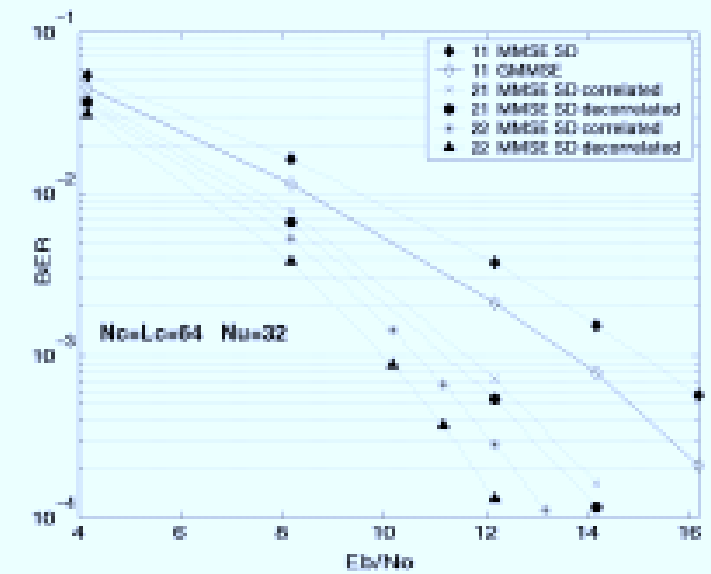

Figure 5. Performance of MMSE MD and SD over METRA channels for SISO $\left(N_{t} N_{r}=11\right)$, MISO $\left(N_{t} N_{r}=21\right)$ and MIMO $\left(N_{t} N_{r}=22\right)$ systems with $N_{c}=L_{c}=64 ; N_{u}=32$.

Figure 5 represents for half load systems $\left(L_{c}=N_{c}=64\right.$ and $\left.N_{u}=32\right)$ and indoor environment the performance of MMSE MD and SD with and without transmit and receive diversity. In the SISO case $\left(N_{t} N_{r}=1-1\right)$, MMSE MD offers, as over Rayleigh channel, a gain of nearly $2 \mathrm{~dB}$ (for a $\mathrm{BER}=10^{-3}$ ) compared to MMSE SD. In the MISO $\left(N_{t} N_{r}=2-1\right)$ and MIMO $\left(N_{t} N_{r}=2-2\right)$ cases, the good performance of the 21 MMSE SD and especially 22 MMSE SD measured over the Rayleigh channel are confirmed when the two channels are perfectly decorrelated. Moreover, in the realistic case corresponding to correlated channels with a $1.5 \lambda$ and $0.4 \lambda$ separation between the two transmit and receive antennas respectively, the performance loss compared to the perfectly decorrelated case is less than $0.5 \mathrm{~dB}$.

\section{CONCLUSION}

The performance of MMSE Single-user Detection (SD) and Multi-user Detection (MD) MIMO MC-CDMA systems are analysed and first compared over Rayleigh fading channels in the case of two transmit antennas and one or two receive antennas. With two transmit antennas and one receive antenna, MD outperforms SD for non-full load systems while the same performance are obtained for full load systems. Besides, with two receive antennas, 22 MMSE SD STBC MC-CDMA offers a very good performance/complexity trade-off. Finally, over the realistic METRA MIMO channel, the very good behaviour of 22 MMSE STBC MC-CDMA systems is confirmed even in the case of correlated channels. 
J-M. AUFFRAY, J-Y. BAUDAIS, J-F. HELARD

\section{ACKNOWLEDGEMENTS}

The authors would like to express their thanks to FTR\&D/DMR/REN which supports this study (contract $\mathrm{n}^{\circ} 42462728$ FT R\&D).

\section{REFERENCES}

[1] S. Hara, R. Prasad, "Overview of multicarrier CDMA", IEEE Communications Magazine, vol.35, N.12, pp. 126-133, December 1997.

[2] V. Tarokh, N. Seshadri, A.R. Calderbank, "Space-time codes for high data rate wireless communication : Performance criterion and code construction", IEEE Transactions on Information Theory, vol. 44, N.2, pp. 744-765, March 1998.

[3] S.M.Alamouti, "A simple transmit diversity technique for wireless communications", IEEE Journal on Selected Areas in Commununications, vol.16, pp. 1451-1458, October 1998.

[4] V. Tarokh, H. Jafarkhani, and A.R. Calderbank, "Space-time block coding for wireless communications: Performance results", IEEE Journal on Selected Areas in Commununications, vol.17, pp. 451-460, March1999.

[5] V. Le Nir, J.M. Auffray, M. Hélard, J.F.Hélard, R. Le Gouable, "Combination of SpaceTime Block Coding with MC-CDMA Technique for MIMO systems with two, three and four transmit antennas", IST mobile \& Wireless Communication Summit, Aveiro, Portugal, June 2003.

[6] S. Kaiser, "Multi-carrier CDMA Radio systems - Analysis and Optimization of Detection, Decoding, and Channel Estimation", PhD. Thesis, VDI-Verlag, Fortschrittberichte VDI, Series 10, N.531,1998.

[7] J-Y. Baudais, J-F. Hélard and J. Citerne, "An improved linear MMSE detection technique for Multi- Carrier CDMA systems : comparaison and combination with interference cancellation", European Transactions on Communications, vol. 11, n 7 , pp. 547-554, November-December 2000.

[8] K.I. Pedersen, J.B. Andersen, J.P. Kermoal, and P.E. Mogensen, "A stochastic MultipleInput-Multiple-Output radio channel model for evluation of space-time coding algorithms", Proceedings of VTC 2000 Fall, pp. 893-897, Boston, United States, September 2000 . 